Revue internationale P.M.E.

Économie et gestion de la petite et moyenne entreprise

\title{
Les travaux publiés dans la Revue internationale PME depuis sa fondation : caractéristiques et tendances
}

\section{Pierre Cossette}

Volume 10, numéro 2, 1997

URI : https://id.erudit.org/iderudit/1009025ar

DOI : https://doi.org/10.7202/1009025ar

Aller au sommaire du numéro

Éditeur(s)

Presses de l’Université du Québec

ISSN

0776-5436 (imprimé)

1918-9699 (numérique)

Découvrir la revue

Citer cette note

Cossette, P. (1997). Les travaux publiés dans la Revue internationale PME depuis sa fondation : caractéristiques et tendances. Revue internationale P.M.E., 10(2), 109-128. https://doi.org/10.7202/1009025ar
Résumé de l'article

Cette étude vise à mettre en relief les caractéristiques et tendances des 112 articles et 29 notes de recherche publiés dans la Revue internationale PME depuis sa fondation en 1988. L'analyse a porté sur les huit aspects suivants : les auteurs, leurs institutions d'attachement et les pays où elles sont situées; les catégories des travaux publiés (articles et notes) ; les types (empirique, conceptuel, méthodologique et épistémologique) auxquels ils peuvent être associés; les échantillons étudiés; les techniques de collecte des données utilisées; les techniques d'analyse des données employées; les références francophones consignées en bibliographie ; et, finalement, les disciplines (économique, stratégie, finance, etc.) auxquelles les travaux peuvent être rattachés. Les résultats sont discutés et quelques voies de recherche sont envisagées. 


\title{
Les travaux publiés dans la Revue internationale PME depuis sa fondation: caractéristiques et tendances ${ }^{1}$
}

Pierre COSSETTE

Université du Québec à Montréal

MOTS CLÉS

Bibliométrie - Publication scientifique

Revue internationale PME

\begin{abstract}
RÉSUMÉ
Cette étude vise à mettre en relief les caractéristiques et tendances des 112 articles et 29 notes de recherche publiés dans la Revue internationale PME depuis sa fondation en 1988. L'analyse a porté sur les huit aspects suivants: les auteurs, leurs institutions d'attachement et les pays où elles sont situées; les catégories des travaux publiés (articles et notes); les types (empirique, conceptuel, méthodologique et épistémologique) auxquels ils peuvent être associés; les échantillons étudiés; les techniques de collecte des données utilisées; les techniques d'analyse des données employées; les références francophones consignées en bibliographie; et, finalement, les disciplines (économique, stratégie, finance, etc.) auxquelles les travaux peuvent être rattachés. Les résultats sont discutés et quelques voies de recherche sont envisagées.
\end{abstract}

1. Une version préliminaire de cet article a été présentée lors du $3^{\mathrm{e}}$ Congrès international francophone de la PME (CIFPME) tenu à Trois-Rivières en 1996. Je tiens à remercier les évaluateurs anonymes pour les précieux commentaires qu'ils avaient alors formulés sur le texte. 


\section{ABSTRACT}

The aim of this study is to highlight the characteristics and trends of the 112 papers and 29 research notes published in Revue internationale PME since it first appeared in 1988. The following eight aspects were analyzed: author, institution and country; category of work published (article or research note) type (empirical, conceptual, methodological, epistemological); samples studied; data collection methods used; data analysis techniques used; French-language references included in the bibliography; and the disciplines (economy, strategy, finance, etc.) covered by the research. The results are discussed and some avenues for further research suggested.

\section{RESUMEN}

La intención de este trabajo es destacar las características y tendencias más relevantes de los 112 artículos y 29 notas de investigación publicados en la Revue internationale PME desde su aparición en 1988. El análisis se ha centrado en los ocho aspectos siguientes: los autores, los centros en los que están y los países donde están situados; la categoría de los trabajos publicados (artículos y notas); los tipos (empírico, conceptual, metodológico y epistemológico) a los que pueden ser asociados; las muestras estudiadas; las técnicas de análisis de datos empleadas; las referencias francófonas consignadas en la bibliografía; finalmente, las disciplinas (economía, estrategia, finanzas, etc.) a las que los trabajos pueden ser adscritos. Se comentan los resultados y se sugieren algunas líneas de investigación.

\section{Problématique et objet de l'étude}

Même si le français est encore une langue internationale, la place qu'il occupe dans le domaine de la publication scientifique est en régression depuis de nombreuses années. L'anglais domine incontestablement, bien que la situation soit moins dramatique dans les sciences sociales et humaines - où le livre occupe une place plus importante et où l'on publie davantage en langue nationale - que dans les sciences naturelles, le génie et les sciences biomédicales (Godin et Vallières, 1995). Plusieurs intervenants francophones (chercheurs, étudiants, décideurs, etc.) déplorent, souvent en faisant preuve d'indignation mais aussi de fatalisme, la disparition graduelle du français comme langue scientifique. Le mouvement serait irréversible.

\section{L'AUTEUR}

Pierre Cossette est professeur à l'École des sciences de la gestion de l'Université du Québec à Montréal. II détient un doctorat en administration de l'Université Laval, ses activités actuelles de formateur et de chercheur témoignent de son intérêt particulier pour les questions d'ordre épistémologique. Adresse : École des sciences de la gestion, Université du Québec à Montréal (UQAM), Case postale 6192, Succursale Centre-ville, Montréal (Québec), H3C 4R2. 
Cette prédominance de l'anglais n'est pas étrangère au fait que les ÉtatsUnis constituent aujourd'hui le centre ou foyer principal de l'activité scientifique (Ben-David, 1971, cité par Godin et Limoges, 1995). Même si, suivant cette référence, ce centre se déplace au fil des siècles et qu'un tel statut fut détenu successivement par l'Italie, l'Angleterre, la France et l'Allemagne, rien ne laisse croire actuellement que la place occupée par l'anglais dans la communication scientifique soit menacée. Au contraire.

Cette « emprise » de la langue anglaise sur la science n'est pas sans inquiéter. Comme la constitution des connaissances a un lien avec la langue du chercheur étant donné que cette dernière lui fournit les mots et les règles à partir desquels il peut penser, on peut craindre l'absence de diversité associée à une science de plus en plus unilingue. Parce que la langue limite ce à quoi on peut penser (Sapir, 1964 ; Whorf, 1969) et que, conséquemment, elle ouvre et ferme inévitablement des portes dans le processus de formation des idées, la production scientifique ne peut être qu'enrichie si elle est l'œuvre de chercheurs qui décodent la réalité à partir d'une langue qui n'est pas toujours la même. Or, les publications scientifiques étant très majoritairement anglophones, le chercheur, actif ou en formation, s'en trouve fortement invité à "penser » en anglais. De plus, dans un tel contexte, il devient difficile pour une communauté scientifique non anglophone de développer et de maintenir des façons de penser et de faire (perspectives, objets, méthodes, etc.) qui lui soient propres. Récemment, Chanlat (1994) a montré que les chercheurs francophones semblent avoir assez bien résisté jusqu'à maintenant au rouleau compresseur anglo-saxon en ce qui a trait à l'analyse organisationnelle ; celle-ci aurait évolué d'une manière particulière dans la francophonie et serait parvenue à conserver ses particularités, dont une tendance à s'éloigner de la position positiviste et fonctionnaliste qui domine les écrits anglophones dans le secteur. Cette autonomie relative serait favorisée, entre autres, par l'existence de revues scientifiques francophones.

Certains se consolent en se disant que la production du savoir doit être distinguée de sa diffusion. Ainsi, le processus de production des connaissances pourrait se dérouler en français et leur diffusion se faire en anglais. Ce raisonnement est dangereux. Le problème ne se limite pas à une simple question de traduction, même s'il ne faut pas oublier qu'elle en est une composante : une traduction ne reflète jamais parfaitement les nuances du texte original. Le problème fondamental en est un d'identité ${ }^{2}$. La communauté scientifique francophone est appelée à être de moins en moins particulière si elle puise uniquement dans les écrits anglophones et si elle ne possède pas de lieux de

2. À ce propos, voir l'éditorial de Julien, Marchesnay et Wtterwulghe (1988) sur la défense de la langue française et l'Institut Pasteur. 
rencontre (par exemple, des revues) à l'intérieur desquels ses membres peuvent faire état de leurs travaux dans une langue qui est la leur. Plus encore, l'effet stimulant de l'existence de tels lieux de partage et de confrontation sur la relève est de nature à contribuer au développement de cette même communauté.

À cet égard, on peut se réjouir de l'existence de revues francophones en sciences de l'administration. On pense, notamment, à Gestion. Revue internationale de gestion, Revue française de gestion et Gestion $2000^{3}$. Bien sûr, elles sont peu nombreuses; elles sont généralement à caractère très général et sont destinées, au moins en partie, au marché des praticiens et des consultants. Mais elles contribuent assurément à alimenter la pensée de différents intervenants francophones dans le champ de l'administration.

Plus rares encore sont les revues francophones dites «scientifiques ou savantes », c'est-à-dire s'adressant prioritairement aux chercheurs désirant apporter une contribution sur le plan conceptuel ou théorique en sciences de l'administration. Parmi celles-ci, il ne semble y en avoir qu'une seule qui soit spécialisée dans le domaine de la PME, soit la Revue internationale PME (RIPME). Bien entendu, son impact international, comme c'est le cas pour l'ensemble des revues québécoises, semble actuellement plutôt limité et son impact national ne serait que légèrement meilleur, si l'on se fie au nombre de citations dont ses articles ont fait l'objet entre 1990 et 1992 (voir Godin et Limoges, 1995). Elle n'en constitue pas moins, avec ses 350 exemplaires de chaque numéro imprimés et distribués un peu partout dans le monde, un véhicule important pour les chercheurs de la communauté scientifique francophone intéressée par la PME. Pour mieux comprendre le succès de cette revue créée il y a moins de 10 ans et pour aider les intervenants concernés à réfléchir sur ce qu'elle pourrait et devrait être dans l'avenir, l'objectif de la présente recherche est de mettre en évidence les caractéristiques et les tendances des articles et des notes de recherche qui y ont été publiés depuis sa fondation en 1988.

\section{Cadre opératoire}

Comme il vient d'être mentionné, je m'en tiendrai ici aux articles et aux notes de recherche, deux productions dont le contenu vise à apporter une contribution scientifique. Les éditoriaux, notes de lecture et informations figurant dans la revue n'ont donc pas été pris en compte.

L'analyse portera essentiellement sur les questions suivantes :

- Qui sont les auteurs, à quelles institutions sont-ils rattachés et de quels pays proviennent-ils?

3. Des articles en anglais sont aussi publiés dans cette dernière revue. 
- Qu' est-ce qui distingue effectivement les articles des notes de recherches?

- Dans quelle mesure les travaux publiés sont-ils de nature empirique, conceptuelle, méthodologique ou épistémologique?

- Comment peut-on caractériser les échantillons auxquels on a eu recours dans les recherches empiriques?

- Comment peut-on caractériser les techniques de collecte des données employées dans les recherches empiriques?

- Comment peut-on caractériser les techniques d'analyse des données utilisées dans les recherches empiriques?

- Les références francophones sont-elles nombreuses dans les textes publiés? Quelle est leur proportion par rapport à l'ensemble des références?

- Dans quelle mesure les travaux publiés ont-ils trait à l'économie ou à la gestion des PME ? Quelles sont les disciplines les plus concernées par ces travaux?

Une attention spéciale sera accordée à l'évolution de toutes ces caractéristiques au fil des ans. Notons que toutes les années ont donné lieu à trois parutions (sauf l'année 1989 où il y en a eu seulement deux), la troisième étant toujours présentée, comme un «bloc» de deux numéros.

\section{Présentation et analyse des résultats}

\subsection{Auteurs, institutions et pays}

Depuis la fondation de la revue en 1988, 217 auteurs, principaux ou secondaires (P ou $\mathbf{S}$ dans les tableaux qui suivent), ont contribué à la production de 112 articles et 29 notes de recherche (A et $\mathbf{N}$ ). Parmi eux se trouvent 185 chercheurs différents : 15 ont contribué en deux occasions, 4 , en trois occasions et 3 , en quatre occasions. Dans la majorité des cas, ceux qui ont contribué plus d'une fois ne l'ont pas fait uniquement comme auteur principal, ni uniquement dans le cas d'articles ; de plus, ils proviennent presque tous du Québec (surtout de l'UQTR) ou de la France.

Les auteurs qui publient dans RIPME ne sont donc pas toujours les mêmes, loin de là. Cette grande diversité - qui ne semble pas particulière à cette revue - laisse voir un intérêt certain chez un nombre impressionnant de chercheurs à publier en français des travaux de recherche présumément inédits sur la PME. Par ailleurs, comme le fait voir le tableau 1, ces chercheurs ont tendance à travailler seul plutôt qu'en collaboration, du moins pour les recherches dont les résultats sont parus dans RIPME. Plus de la moitié des articles publiés 
jusqu'à maintenant dans cette revue ont un auteur unique et cette tendance est encore plus forte pour les notes de recherche. Notons que ce niveau de collaboration entre chercheurs (en supposant qu'elle existe lorsqu'il y a plusieurs auteurs) a très peu varié depuis 1988 ; cependant, depuis 1991, les textes publiés par deux auteurs sont plus nombreux à chaque année, exception faite des années 1992 et 1993 où leur nombre fut le même $(n=5)$. En tout, $41 \%$ des textes publiés dans RIPME sont cosignés, ce qui est très supérieur au taux de cosignature de $23,1 \%$ des articles publiés dans les revues québécoises entre 1990 et 1992 dans l'ensemble des sciences sociales et humaines (Godin et Limoges, 1995).

La provenance de ces auteurs, tant en ce qui a trait aux établissements auxquels ils se disent rattachés qu'aux pays où sont situés ces établissements, est tout simplement remarquable par sa diversité, même si les établissements universitaires du Québec et de la France se détachent très nettement des autres à ces égards. En huit ans d'existence, la revue a reçu des contributions provenant de 95 établissements (universitaires pour la plupart) situés dans 21 pays différents. Cette situation mérite d'être examinée de plus près.

Le tableau 2 met en évidence les établissements ayant le plus contribué à RIPME. On constate d'abord que les sept institutions les plus "productives » sont du Québec (4) et de la France (3). Ensuite, on remarque que la contribution de l'UQTR, en plus d'être dans une classe à part par son ampleur, paraît grandissante depuis 1993, particulièrement en ce qui touche le nombre d'auteurs secondaires issus de cette institution. L'Université Laval effectue un retour en force depuis 1994 et les universités de Nice-Sophia Antipolis et Lumière-Lyon II ont fourni chacune trois auteurs principaux d'un article depuis 1993. Parallèlement, les contributions de l'UQAM, de l'Université de Montpellier I et de l'École polytechnique de Montréal semblent en décroissance. Il est également intéressant de noter la très forte tendance des chercheurs provenant de ces 7 institutions à contribuer (à titre d'auteur principal ou secondaire) à la production d'articles plutôt que de notes de recherche. Finalement, il faut signaler que 55 des 95 établissements contributeurs n'ont fourni qu'un seul auteur ou moins ${ }^{4}$.

Les pays dont les institutions ont fourni la plus grande contribution à RIPME sont présentés dans le tableau 3. On ne se surprendra guère que la France et le Québec ${ }^{5}$ se détachent nettement des autres pays. Deux observations

4. L'évaluation de la contribution d'une institution s'est faite à partir du nombre d'établissements auxquels chaque auteur disait être rattaché. Ainsi, cette évaluation a été de 0,5 par établissement dans les quelques cas où un auteur reconnaissait être affilié à deux institutions.

5. Pour des raisons sociopolitiques, notamment, le Québec est ici considéré comme distinct du Canada. 
TABLEAU I

Nombre d'articles et de notes de recherche

en relation avec le nombre d'auteurs

\begin{tabular}{|c|c|c|c|c|c|c|c|c|c|c|c|c|c|c|c|c|c|c|}
\hline & \multicolumn{2}{|c|}{1988} & \multicolumn{2}{|c|}{1989} & \multicolumn{2}{|c|}{1990} & \multicolumn{2}{|c|}{1991} & \multicolumn{2}{|c|}{1992} & \multicolumn{2}{|c|}{1993} & \multicolumn{2}{|c|}{1994} & \multicolumn{2}{|c|}{1995} & \multicolumn{2}{|c|}{ Total } \\
\hline & $\mathbf{A}$ & $\mathbf{N}$ & $\mathbf{A}$ & $\mathbf{N}$ & $\mathbf{A}$ & $\mathbf{N}$ & $\mathbf{A}$ & $\mathbf{N}$ & $\mathbf{A}$ & $\mathbf{N}$ & $\mathbf{A}$ & $\mathbf{N}$ & $\mathbf{A}$ & $\mathbf{N}$ & $\mathbf{A}$ & $\mathbf{N}$ & $\mathbf{A}$ & $\mathbf{N}$ \\
\hline 1 auteur & 7 & - & 11 & 1 & 9 & 1 & 6 & 4 & 11 & 3 & 6 & 3 & 7 & 3 & 6 & 5 & 63 & 20 \\
\hline 2 auteurs & 5 & 1 & 2 & 1 & 4 & - & 2 & 1 & 5 & - & 4 & 1 & 5 & 1 & 9 & 2 & 36 & 7 \\
\hline 3 auteurs & 2 & - & - & - & 3 & - & 1 & - & - & - & 2 & - & 2 & 1 & - & - & 10 & 1 \\
\hline 4 auteurs & - & - & - & - & - & - & I & - & - & - & - & 1 & 1 & - & 1 & - & 3 & 1 \\
\hline Total & 14 & 1 & 13 & 2 & 16 & 1 & 10 & 5 & 16 & 3 & 12 & 5 & 15 & 5 & 16 & 7 & 112 & 29 \\
\hline
\end{tabular}


Tableau 2

Établissements ayant le plus contribué à la Revue internationale PME

(ayant fourni au moins trois auteurs principaux d'un article)

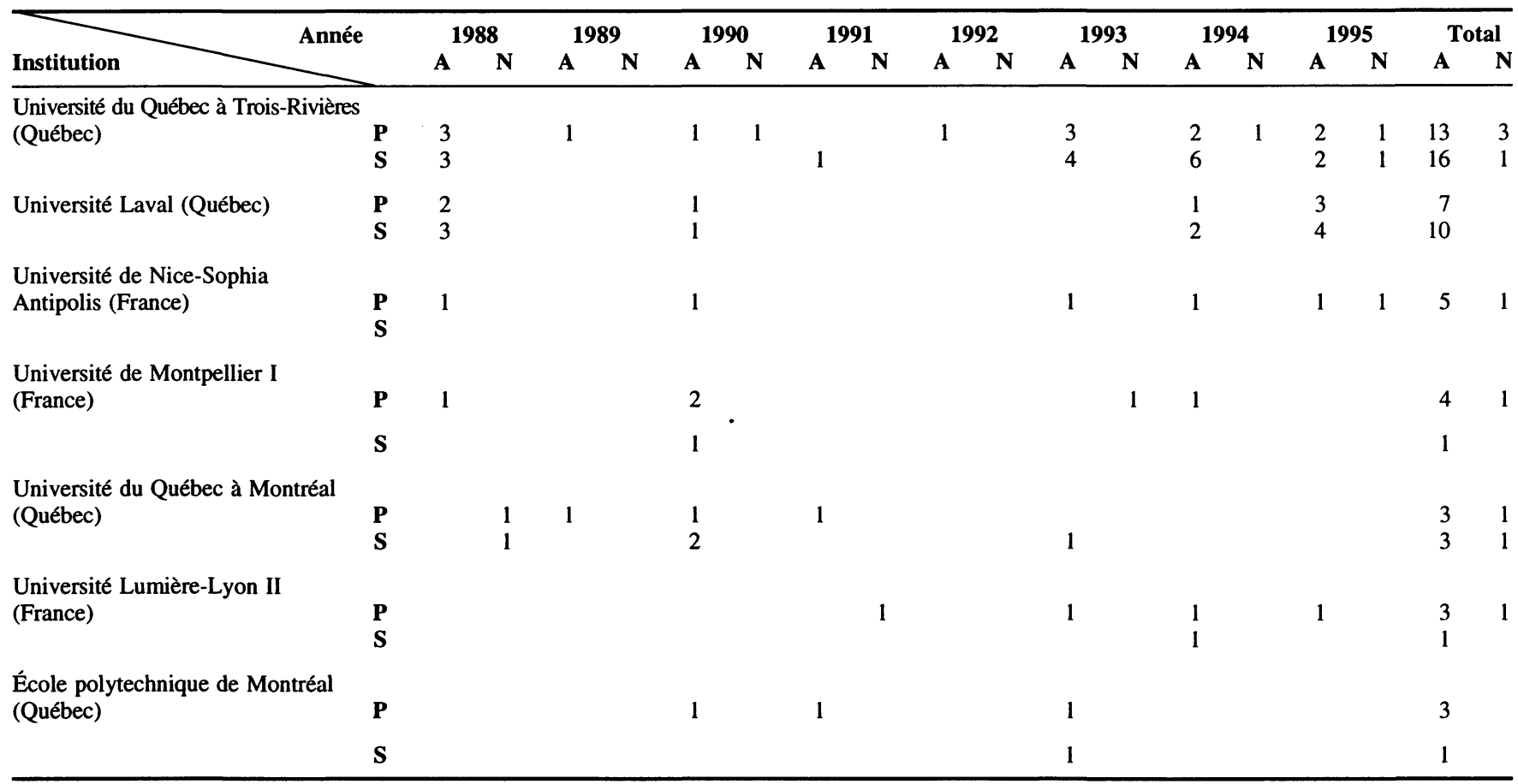




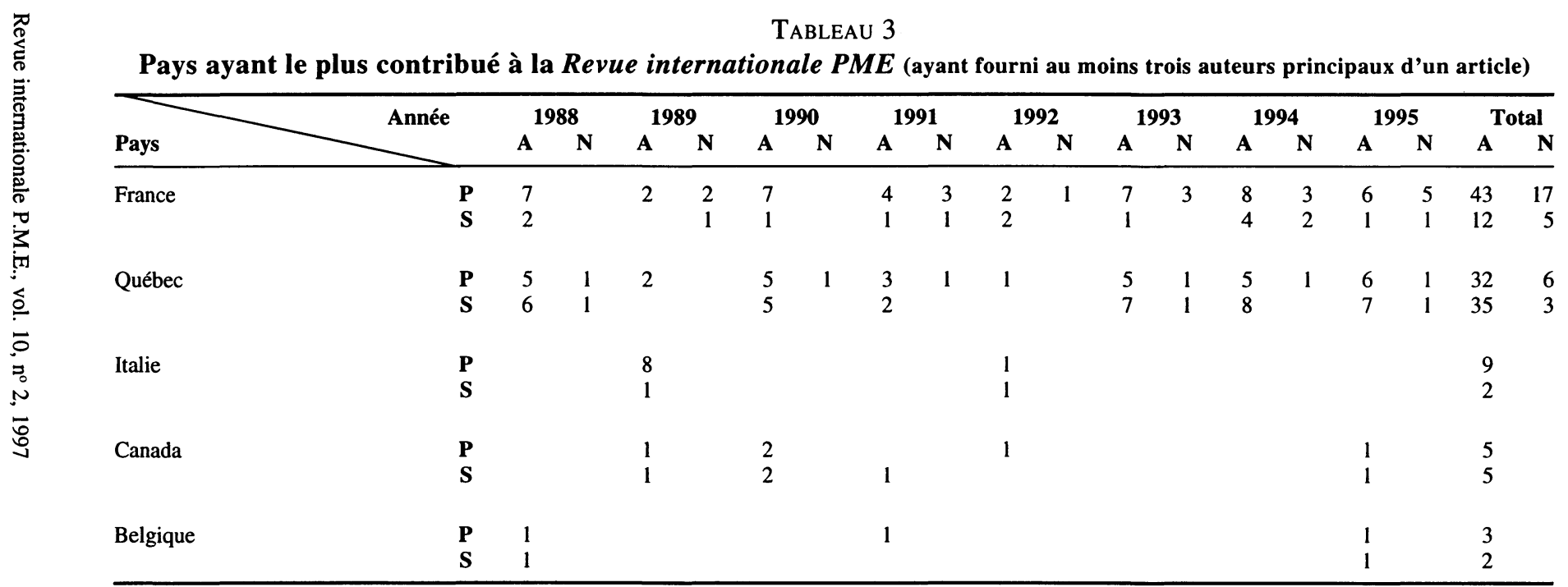


peuvent être faites ici. D'abord, la forte contribution de l'Italie s'explique par la parution en 1989 d'un numéro spécial sur les districts italiens dans lequel huit auteurs principaux et un auteur secondaire provenaient de ce pays. Ensuite, les contributions canadiennes et belges ont été marginales respectivement depuis 1993 et 1992. Par ailleurs, si l'on tient compte de l'ensemble des 21 pays, on note que 5 d'entre eux n'ont qu' une seule contribution à RIPME, mais qu'elle a eu lieu dans tous les cas depuis 1992, ce qui laisse penser que la revue s'internationalise encore davantage. Ajoutons que l'apport de l'Espagne, inexistant jusqu'en 1993, semble en pleine croissance depuis ce temps.

En somme, ce qui caractérise les auteurs, les institutions auxquelles ils sont rattachés et les pays où ils œuvrent, c'est d'abord leur grande variété. Cette diversité est tellement grande qu'elle peut laisser une impression de «fragmentation ", d'isolement même. À part quelques exceptions, rien ne permet de croire en l'existence de liens particulièrement étroits entre les chercheurs qui publient dans RIPME ou entre les institutions et les pays d'où ils proviennent.

\subsection{Catégories}

Comme il a déjà été mentionné, 112 articles et 29 notes de recherche ont été publiés dans RIPME depuis sa fondation. Le nombre d'articles varie relativement peu d'une année à l'autre, mais le nombre de notes de recherche a augmenté considérablement depuis 1993 ; en effet, 17 des 29 notes sont parues au cours des trois dernières années. Le statut d' "article » est-il maintenant plus difficile à atteindre parce que les exigences seraient devenues plus grandes ? Signalons également que, contrairement aux articles, les notes sont plus souvent à caractère conceptuel $(n=15)$ que de nature empirique $(n=11)$. Cette tendance constituet-elle une orientation plus ou moins explicite donnée à la revue et, si tel est le cas, n'est-ce pas un peu inquiétant?

Dans l'éditorial du premier numéro de la revue (septembre 1988), le comité de rédaction écrivait qu'aux articles s' « ajouteront des notes plus courtes par exemple sur des analyses de cas pouvant permettre d'améliorer la théorie en développement ${ }^{6} \gg$ (p. 5). Qu'en est-il exactement ? Dans les faits, on constate rapidement que la longueur d'un texte est un critère qui, dans l'ensemble, n'a pas vraiment permis jusqu'à maintenant de distinguer les articles des notes de recherche. Par exemple, d'une part, 18 articles (16\% du nombre total, même

6. Selon Pierre-André Julien, directeur de la revue, des travaux intéressants mais exploratoires ou pas tout à fait au point, ou encore des textes à propos desquels les évaluateurs ont émis des avis qui s'opposent, peuvent se voir attribuer le statut de «note de recherche» (Communication personnelle, 1996). 
s'il n'y en a eu que deux depuis 1992) et seulement sept notes (24\% du nombre total, dont cinq depuis 1993) comprennent 15 pages ou moins; d'autre part, cinq notes (17\%), dont deux en 1995, occupent 25 pages ou plus. Par ailleurs, il semble que les articles soient de plus en plus longs. Depuis 1993, 35 d'entre eux couvrent 25 pages ou plus. Toutes ces constatations laissent penser que les textes les plus longs et les textes les plus courts ont de plus en plus tendance à devenir respectivement des articles et des notes.

Les notes constituent-elles surtout des «analyses de cas», comme le suggérait l'éditorial mentionné plus haut? Les informations recueillies ne permettent pas de l'affirmer, d'autant plus que les rares analyses de cas font parfois l'objet d'articles plutôt que de notes. Cependant, tout peut dépendre de ce qu'on entend par "analyse de cas». Le plus souvent, une analyse de cas est associée à une étude en profondeur faite sur un petit échantillon (une seule situation ou un seul sujet le plus couramment) et se fondant sur un ensemble de données particulières recueillies à l'aide de méthodes reconnues et analysées de manière rigoureuse.

\subsection{Types}

Les distinctions entre les différents types de recherche ne sont pas faciles à établir. Dans la présente étude, j'ai considéré qu'il y avait quatre types de recherche : empirique, lorsqu'il s'agit d'une recherche sur le terrain ou réalisée à partir d'informations provenant d'une banque de données ; conceptuel, lorsqu'il s'agit d'une réflexion ou d'une analyse portant sur un thème particulier et où les données chiffrées, s'il en existe, ne viennent qu'illustrer ou appuyer un aspect de la réflexion; méthodologique, lorsque le travail possède un caractère clairement instrumental, associé à la production des connaissances ou à l'intervention ; et épistémologique, quand l'objet fondamental du travail est d'étudier les travaux d'autres chercheurs en vue d'en déterminer les caractéristiques.

Le tableau 4 montre l'évolution des types d'articles et de notes publiés dans RIPME depuis sa fondation. D'entrée de jeu, on voit que les recherches empiriques dominent, suivies des recherches à caractère conceptuel et, loin derrière, des travaux de nature méthodologique ou épistémologique. Deux observations doivent être faites : d'abord, chacun de ces types a un poids non négligeable qui met en relief une variété certaine - une ouverture diront certains - qu'on retrouve dans peu de revues savantes; ensuite, cette variété est remarquablement stable dans le temps. 
TABleau 4

Types d'articles et de notes publiés dans la Revue internationale PME

\begin{tabular}{lccccccccc}
\hline & 1995 & 1994 & 1993 & 1992 & 1991 & 1990 & 1989 & 1988 & Total \\
\hline Empirique & 12 & 14 & 8 & 8 & 6 & 11 & 4 & 10 & 73 \\
Conceptuel & 10 & 5 & 5 & 10 & 8 & 4 & 10 & 3 & 55 \\
Méthodologique & - & - & 2 & 1 & 1 & 1 & - & 2 & 7 \\
Épistémologique & 1 & 1 & 2 & - & - & 1 & 1 & - & 6 \\
\hline
\end{tabular}

Un examen attentif des données qui ont permis de dresser ce tableau fait voir, notamment, que les chercheurs associés aux textes méthodologiques et épistémologiques ont tendance à travailler davantage en collaboration que les autres : quatre sur sept dans le premier cas et trois sur six dans le second. Aussi, les travaux d'ordre méthodologique ont très nettement un lien direct avec la stratégie et les systèmes d'information organisationnels, alors que les travaux d'ordre épistémologique sont plutôt orientés du côté de la gestion des ressources humaines et de l'économique.

\section{4. Échantillons}

La taille des échantillons varie beaucoup. Plusieurs recherches sont des études détaillées de seulement quelques individus, entreprises ou secteurs. La majorité des travaux comptent entre 25 et 100 sujets. Une douzaine ont un échantillon de plus de 400 individus et au moins huit ont porté sur l'étude de plus de 1000 personnes ou entreprises. Les propriétaires-dirigeants ou entrepreneurs ${ }^{7}$ sont évidemment les plus sollicités. Cette diversité se manifeste également dans les entreprises étudiées. Elles représentent un grand nombre de secteurs économiques et proviennent de la multitude de pays dont il fut question précédemment.

\subsection{Techniques de collecte des données}

D'abord, il faut malheureusement noter que, trop souvent, les techniques employées pour la collecte des données ne sont pas précisées clairement. Par exemple, on ne sait pas toujours si les questions posées sont fermées, à éventail de réponses, à évaluation (type « échelle de Likert ») ou ouvertes. Parfois même, on se demande si le questionnaire a été posté ou si les sujets ont été rencontrés par le chercheur. Ces remarques invitent à la réflexion, surtout lorsque l'on considère que dans l'étude de Godin et Limoges (1995), les revues québécoises en sciences sociales

7. La distinction entre ces deux concepts est rarement prise en considération, même s'ils ne devraient pas être confondus (Wortman, 1986). 
et humaines ont été jugées sous le niveau moyen pour ce qui est du critère de qualité «méthode» par les 689 professeurs de ce secteur qui avaient accepté de participer à l'enquête.

Le questionnaire est la technique à laquelle les chercheurs ont le plus souvent recours. Généralement, les sujets le reçoivent par la poste ou y répondent dans le cadre d'une entrevue : un peu plus de 25 recherches ont été réalisées suivant le premier mode et autant suivant le second. Les questions les plus fréquemment employées (lorsque cette information est clairement précisée) sont, dans l'ordre, fermées, à évaluation, à éventail de réponses et ouvertes. L'entrevue en profondeur, l'observation (plus ou moins participante) et l'utilisation de banques de données, trois approches très peu employées avant 1993, mais qui le sont de plus en plus; en leur reconnaissant ainsi une légitimité à laquelle d'autres revues scientifiques semblent encore réticentes à souscrire, surtout en ce qui a trait aux deux premières, RIPME fait preuve d'une ouverture grandissante ${ }^{8}$ qui pourrait ne pas faire l'unanimité.

\subsection{Techniques d'analyse des données}

Là encore, on doit déplorer que les techniques d'analyse employées ne soient pas toujours clairement précisées, même si l'occurrence de ce problème est plus faible que dans le cas des techniques de collecte des matériaux de la recherche. Parfois même, l'analyse des résultats ressemble à une discussion dont le fondement est plutôt flou. Par ailleurs, à la lumière de ce qui a été dit dans la section précédente, on ne s'étonne pas que l'analyse des résultats soit beaucoup plus «quantitative » que «qualitative». À titre illustratif, l'analyse de contenu n'a été employée qu'en quelques occasions.

En ce qui a trait aux statistiques utilisées, on constate d'abord la forte présence de statistiques descriptives simples qui fournissent des indices de position ou de dispersion souvent intéressants : moyennes, écarts types, rangs, pourcentages, etc. Ensuite, on voit que, en excluant 1995, c'est en 1988 que les statistiques furent le plus utilisées pour analyser les matériaux des travaux publiés ; il n'est pas impossible que, à l'origine, les dirigeants de RIPME aient eu plus ou moins explicitement la conviction que la crédibilité de la revue serait plus facilement établie si les conclusions des recherches reposaient sur une argumentation quantitative. Étonnamment, on constate, dans les textes des deux premiers numéros publiés en 1995 (c'est-à-dire en excluant le numéro

8. Signalons toutefois que l'entrevue en profondeur et l'observation ont donné lieu principalement à des notes de recherche plutôt qu'à des articles. 
spécial portant sur le financement des PME), une utilisation massive de statistiques qui dépasse nettement ce qu'on observait dans les années antérieures, y compris en 1988. Il y a peut-être là un simple effet du hasard...

Les techniques multivariées sont largement employées dans les recherches publiées dans RIPME, et de façon plutôt stable au fil des ans. Parmi celles qui ont pour objectif de « décrire», l'analyse factorielle est de loin la plus populaire : son utilisation est à peu près deux fois plus fréquente que toutes les autres réunies, incluant l'analyse discriminante et l'analyse de regroupement. Quant aux techniques multivariées visant à «expliquer », donc associées à l'inférence statistique, leur utilisation est beaucoup moins fréquente : l'analyse de régression n'a été employée qu'en quelques occasions ${ }^{9}$, toutes cependant depuis 1992 , et l'analyse de variance n'a été employée apparemment qu'une seule fois, en 1988.

\subsection{Références francophones}

Très clairement, comme le montre le tableau 5, les travaux des chercheurs francophones intéressés par la PME sont connus et reconnus. Ou peut-être serait-il plus juste d'affirmer que, au moins dans RIPME et pour diverses raisons, certains travaux de certains chercheurs ont un certaine notoriété... L'étude de ces aspects particuliers serait sûrement pertinente, mais dépasse le cadre de la présente recherche.

TABleau 5

Références francophones dans les articles et notes publiés dans la Revue internationale PME

\begin{tabular}{llllllllll}
\hline & 1995 & 1994 & 1993 & 1992 & 1991 & 1990 & 1989 & 1988 & Moyenne \\
\hline $\begin{array}{l}\text { Nombre total } \\
\text { Nombre moyen }\end{array}$ & 303 & 291 & 203 & 195 & 250 & 203 & 102 & 177 & 215,5 \\
$\quad$ par article ou note & 13,2 & 14,6 & 11,9 & 10,3 & 16,7 & 11,9 & 6,8 & 11,8 & 12,2 \\
Pourcentage moyen & 42,2 & 42,7 & 38,1 & 53,2 & 51,3 & 60,8 & 31,2 & 50,5 & 46,3 \\
\hline
\end{tabular}

Les articles et notes des trois numéros de RIPME généralement publiés annuellement contiennent en moyenne un total d'un peu plus de 200 références francophones, soit 12,2 en moyenne par texte. Aucune tendance particulière ne semble se manifester dans l'évolution annuelle de ces chiffres, si ce n'est une légère augmentation de ces deux moyennes depuis 1994 ; les productions

9. À cet égard, il convient de noter que Bygrave et Hofer (1991) suggèrent de délaisser l'analyse de régression : «[...] regression analysis is reductionist, while entrepreneurship is holistic [it] assumes stable models built with relatively few variables, rather than unstable models with many variables » (p. 20). 
francophones sont peut-être plus nombreuses, de meilleure qualité ou mieux connues qu'auparavant, ce qui pourrait expliquer, au moins en partie, qu'on y fasse davantage référence. Par ailleurs, beaucoup d'auteurs citent leurs propres travaux en français, parfois exagérément prétendront certains, même lorsqu'il s'agit de cahiers de recherche, de documents de travail ou d'autres productions non publiées. Finalement, le tableau 5 montre que le pourcentage de références francophones est presque égal au pourcentage de références non francophones (principalement anglophones, mais également italiennes, espagnoles, etc.), et que ce pourcentage fluctue relativement peu dans le temps, malgré une certaine baisse depuis 1993 .

Terminons cette section avec quelques observations ayant trait uniquement aux textes contenant $90 \%$ ou plus de références francophones, ou $10 \%$ ou moins :

- sur les 16 textes de la première catégorie et les 25 de la seconde, il n'y a que deux et trois notes de recherche, respectivement ;

- il y a très peu d'auteurs du Québec, dans un cas comme dans l'autre;

- il n'y a eu que deux textes depuis 1993 contenant $90 \%$ ou plus de références francophones ;

- les textes dont $90 \%$ ou plus des références sont francophones n'ont jamais plus de deux auteurs et portent surtout sur l'économique et la finance ;

- les textes qui ont $10 \%$ ou moins de références francophones sont majoritairement à caractère conceptuel et ont surtout trait à l'économique (plus de la moitié) et à la stratégie.

\subsection{Disciplines}

Il est parfois très difficile d'associer une recherche à une discipline particulière. Le découpage même du champ des sciences de l'administration en divers souschamps et les frontières de ces sous-champs ne font pas l'unanimité. Ajoutons que plusieurs objets de recherche semblent relever de plus d'une discipline... Dans chaque numéro de RIPME, on trouve les indications suivantes, relativement à la spécificité de la revue:

La revue vise à promouvoir la diffusion [...] de recherches concernant l'économie et la gestion des petites et moyennes entreprises (PME). Par économie, on entend, entre autres, des études sur le rôle et la place de la PME dans le développement national et régional ou encore sur la relation entre la PME et son environnement économique. Par gestion, on pense à des recherches sur les comportements de la PME et sur les différentes fonctions de gestion telles que la stratégie, le marketing, la finance, les ressources humaines, l'information, etc. 
Ces précisions, y compris le découpage partiel qu'elles impliquent, ont été retenues ici et, finalement, neuf catégories ont été déterminées. Le tableau 6 présente le nombre de textes auxquels chacune de ces disciplines peut être associée depuis 1988. Étant donné que ce découpage des sciences de l'administration en neuf disciplines et que l'attribution de chaque article et de chaque note à une ou deux disciplines sont fondés sur des jugements qui ne feraient pas nécessairement consensus parmi les membres de notre communauté scientifique, les résultats qui suivent doivent être interprétés avec prudence.

TABLEAU 6

Disciplines auxquelles sont rattachés les articles et notes publiés dans la Revue internationale PME

\begin{tabular}{lrrrrrrrrr}
\hline & 1995 & 1994 & 1993 & 1992 & 1991 & 1990 & 1989 & 1988 & Total \\
\hline Économique & 12 & 9 & 8 & 12 & 6 & 3 & 13 & 3 & 66 \\
Management & 4 & 5 & 4 & 3 & 5 & 3 & 1 & 5 & 30 \\
Stratégie & 1 & 8 & 4 & - & 3 & 3 & - & 3 & 22 \\
$\begin{array}{l}\text { Finance } \\
\begin{array}{l}\text { Comportement } \\
\quad \text { organisationnel }\end{array}\end{array}$ & 10 & - & 1 & 4 & 2 & 1 & 1 & - & 19 \\
$\begin{array}{l}\text { Gestion } \\
\quad \text { des ressources }\end{array}$ & & & - & - & 1 & 2 & - & 4 & 12 \\
$\quad$ humaines & - & 1 & 2 & 1 & - & 6 & - & - & 10 \\
$\begin{array}{l}\text { Système } \\
\quad \text { d'information }\end{array}$ & & & & & & & & & \\
$\quad$ organisationnel & 1 & - & 2 & - & 1 & - & 1 & 3 & 8 \\
$\begin{array}{l}\text { Marketing } \\
\text { Gestion }\end{array}$ & 1 & - & - & - & - & 1 & - & 1 & 3 \\
$\quad$ des opérations & - & 2 & - & - & - & - & - & - & 2 \\
\hline
\end{tabular}

Les données du tableau 6 vont tout à fait dans les deux grandes directions mentionnées plus haut. Près de la moitié des textes ont un lien direct avec l'économique. De plus, l'équilibre entre les recherches d'orientation «macro " (économique) et d'orientation «micro » (gestion) semble se maintenir au fil du temps. Le tandem management-stratégie domine fortement les recherches reliées à la gestion des PME, et cette tendance va en s'accentuant: la moitié des textes de cet ensemble ont été publiés depuis 1993. La gestion des opérations et le marketing n'occupent qu'une place marginale. Quelques remarques peuvent aussi être faites à propos du lien entre, d'une part, la discipline concernée et, d'autre part, la catégorie et le type de recherche : 
- 17 des 29 notes de recherche relèvent, au moins partiellement, de l'économique ;

- aucun texte associé à la finance ou à la gestion des ressources humaines n'a donné lieu à une note de recherche, et les deux seuls textes associés à la gestion des opérations ont été des notes de recherche ;

- 44 des 55 textes à caractère conceptuel ont un lien direct avec l'économique ;

- les sept recherches de type méthodologique sont directement associées à la stratégie, aux systèmes d'information organisationnels ou au management, alors que les six recherches de type épistémologique sont associées à cinq disciplines différentes.

\section{Conclusion}

C'est probablement la diversité - qui ne se dément pas au fil des ans - qui caractérise le mieux cette revue savante qu'est RIPME. Cette variété se manifeste dans les auteurs, les institutions d'où ils proviennent et les pays représentés ${ }^{10}$. Elle est aussi très en évidence dans les types de recherche qui y sont publiés (empirique, conceptuel, méthodologique et épistémologique) et dans les échantillons constitués (nombre de sujets, secteurs concernés, etc.). Les références francophones sont fort nombreuses, mais les références non francophones le sont également. Les articles et notes publiés ont trait presque également à l'économie des PME et à leur gestion. Parfois même, cette diversité est si grande qu'elle peut laisser une impression de «fragmentation» tellement il semble y avoir peu de rapports entre les recherches ${ }^{11}$. Churchill et Lewis (1986) avaient aussi noté cette même tendance à la fragmentation dans les pistes de recherche empruntées par les chercheurs en PME. La multiplication de numéros thématiques (cinq depuis 1992) peut vraisemblablement contribuer à réduire cette impression de fragmentation.

10. Tout indique que RIPME peut effectivement être dite « internationale», au moins dans la francophonie, non seulement par la provenance des auteurs, mais également par celle des membres du comité de rédaction et du comité scientifique. Elle semble même s'internationaliser de plus en plus.

11. J'avais déjà tiré la même conclusion d'une recherche à propos des objets cognitifs dans la littérature scientifique sur la PME et l'entrepreneuriat (Cossette, 1995) et Déry (1989) avait fait de même relativement à la problématique de la décision dans la revue ASQ. Plus récemment, Déry et Toulouse (1996) concluaient, eux aussi, à la suite de leur étude des 237 articles parus dans Journal of Business Venturing entre 1986 et 1993 que la fragmentation (particulièrement sociale) caractérisait fortement le champ de recherche de l'entrepreneuriat. 
Les techniques de collecte et d'analyse des données employées dans les travaux publiés autorisent à penser que les auteurs se placent dans une perspective plutôt traditionnelle et associée au modèle orthodoxe de production des connaissances ${ }^{12}$, même si cette orientation semble de moins en moins dominante. Les résultats reposent le plus souvent sur des données obtenues en réponse à des questions fermées, à éventail de réponses et à évaluation ; une telle approche est «structurante» en ce sens qu'elle fournit au sujet un cadre de référence qui n'est pas le sien, mais celui du chercheur ${ }^{13}$. L'analyse, réalisée alors à l'aide de techniques quantitatives, est généralement à visée nomothétique.

Même si certains des résultats obtenus ici ont pu être mis en rapport avec ceux fournis par l'étude de Godin et Limoges (1995), les caractéristiques et tendances des travaux publiés dans RIPME peuvent difficilement être comparées à celles des travaux publiés dans d'autres revues en sciences de l'administration parce qu'il n'y a à peu près pas de données à ce sujet. De telles comparaisons aideraient pourtant à mieux distinguer RIPME de ses concurrents (principalement francophones mais aussi anglophones) et des autres revues scientifiques en administration, ainsi qu'à mettre en relief l'identité particulière de cette revue. Dans cette même veine, une voie de recherche intéressante serait de comparer les objets étudiés par les chercheurs dans RIPME à ceux abordés dans les revues professionnelles ou s'adressant principalement aux praticiens de la PME $^{14}$. Quels sont les objets privilégiés dans les deux cas ? L'intérêt exprimé pour différents objets dans RIPME précède-t-il ou suit-il celui affiché dans les revues professionnelles?

Dans la présente recherche, l'étude de ces objets ou questions de recherche à la base des articles et notes publiés dans RIPME (ainsi que leur évolution dans le temps) aurait certainement été fort intéressante et enrichissante, notamment en fonction d'une éventuelle comparaison de RIPME avec d'autres revues... mais elle aurait également été très exigeante. Il y a là matière à projet de recherche pour le chercheur intéressé par les questions d'ordre épistémologique. Il en est de même de l'étude plus approfondie de certains aspects abordés ici. Par exemple, en ce qui a trait aux références francophones, qui sont les auteurs les plus cités? Quels sont les textes francophones les plus cités ? S'agit-il d'articles de revues savantes (lesquelles?), d'articles de revues professionnelles (lesquelles?), d'actes de colloque, de documents de travail, de rapports particuliers? etc. Les références en français sont-elles récentes?

12. Pour une critique de ce modèle, voir notamment Audet, Landry et Déry (1986).

13. À ce propos, voir notamment Cossette (1994).

14. Sur cette question, voir entre autres Ireland et Van Auken (1987), Brockhaus (1987) ainsi que Sandberg et Gatewood (1991). 
Quel est le pourcentage d'autocitations ? À cet égard, le chercheur intéressé aura avantage à regarder de très près la démarche empruntée par Déry et Toulouse (1996) pour étudier les articles (en particulier, les réseaux qui émergent d'une analyse des textes cités par les auteurs de ces articles) publiés dans Journal of Business Venturing de 1986 à 1993.

Cette revue, qui appartient en quelque sorte à tous les chercheurs francophones œuvrant dans le domaine de la PME, a connu du succès jusqu'à présent et rien ne laisse croire qu'elle n'en connaitra pas davantage dans l'avenir. RIPME est peut-être même devenue une revue suffisamment «mature» pour se permettre d'être encore plus exigeante en ce qui a trait à la qualité des textes qu'elle accepte de publier. Par exemple, les caractéristiques des techniques de collecte et d'analyses de données utilisées (et peut-être aussi celles des échantillons) devraient, dans tous les cas, être très clairement précisées. Les résultats de cette recherche ont mis en évidence certaines caractéristiques et tendances des articles et notes publiés dans RIPME. Souhaitons qu'ils contribuent à alimenter nos réflexions, en particulier celles du comité de direction de la revue, sur ce que pourrait et devrait être notre revue dans les prochaines années.

\section{Bibliographie}

AUDET, M., M. LANDRY et R. DÉRY (1986), « Science et résolution de problème : liens, difficultés et voies de dépassement dans le champ des sciences de l'administration ", Philosophy of the Social Sciences / Philosophie des sciences sociales, vol. 16, p. 409-440.

Ben-DAvid, J. (1971), The Scientist's Role in Society : A Comparative Study, Chicago, University of Chicago Press.

BROCKHAUS, R.H., Sr. (1987), «Entrepreneurial research : are we playing the correct game? », American Journal of Small Business, vol. 11, n 3, p. 43-49.

BYGRAVE, W.D. et C.W. HOFER (1991), "Theorizing about entrepreneurship», Entrepreneurship Theory and Practice, vol. 16, $\mathrm{n}^{\circ}$ 2, p. 13-22.

Chanlat, J.-F. (1994), "Francophone organizational analysis (1950-1990): an overview », Organization Studies, vol. 15, no 1, p. 47-79

ChURChILL, N.C. et V.L. LEWIS (1986), «Entrepreneurship research : directions and methods ", p. 333-365 dans D.L. Sexton et R.W. Smilor (dir.), The Art and Science of Entrepreneurship, Cambridge, MA, Ballinger.

COSSETTE, P. (1994), «Les cartes cognitives au service de l'étude des organisations », dans P. Cossette (dir.), Cartes cognitives et organisations, Québec, Les Presses de l'Université Laval, collection « Sciences de l'administration »; Paris, Éditions Eska, p. 3-12. 
Cossette, P. (1995), « La cognition comme objet d'étude dans la littérature scientifique sur la PME et l'entrepreneuriat », dans Actes du $2^{e}$ Congrès international francophone de la PME (CIFPME), Paris, p. 487-524.

DÉRY, R. (1989), La structuration discursive de la problématique de la décision dans la revue Administration Science Quarterly: une contribution à l'épistémologie des sciences de l'organisation, Thèse de doctorat inédite, Québec, Université Laval.

DÉRY, R. et J.-M. TOULOUSE (1996), «Social structuration of the field of entrepreneurship : a case study », Canadian Journal of Administrative Sciences /Revue canadienne des sciences de l'administration, vol. 13, $\mathrm{n}^{0} 4$, p. 285-305.

GodiN, B. et C. LIMOGES (1995), Les revues scientifiques québécoises : une évaluation du programme de soutien aux revues du Fonds FCAR, Étude réalisée pour le Fonds FCAR, Québec.

Godin, B. et F. VALliÈres (1995), Une nouvelle estimation de la part de français dans les communications scientifiques, Étude réalisée pour le Conseil de la langue française et du ministère de l'Industrie, du Commerce, de la Science et de la Technologie, Québec.

IRELAND, R.D. et P.M. VAN AUKEN (1987), «Entrepreneurship and small business research : an historical typology and directions for future research », American Journal of Small Business, vol. 11, $\mathrm{n}^{\circ} 4$, p. 9-20.

JULIEN, P.-A., M. MARCHESNAY et R. WTTERWULGHE (1988), «La défense de la langue française et l'Institut Pasteur » (éditorial), Revue internationale PME, vol. 1 , $\mathrm{n}^{\text {os }} 3-4$, p. 255-257.

SANDBERG, W.R. et E.J. GATEWOOD (1991), «A profile of entrepreneurship research centers : orientations, interests, activities, and resources », Entrepreneurship Theory and Practice, vol. 15, $\mathrm{n}^{\circ} 3$, p. 11-24.

SAPIR, E. (1964), Culture, Language and Personality, Berkeley, University of California Press.

WhORF, B. (1969), Linguistique et anthropologie, Paris, Denoël-Gonthier.

WORTMAN, M.S., Jr. (1986), «A unified framework, research typologies, and research prospectuses for the interface between entrepreneurship and small business », dans D.L. Sexton et R.W. Smilor (dir.), The Art and Science of Entrepreneurship, Cambridge, MA, Ballinger, p. 273-331. 